\title{
Comparison of Quality of Life Between Iranians Using Two Implant-Retained Overdenture Prosthesis and Conventional Complete Denture
}

\author{
Hassan Semyari ${ }^{1}$; Majideh Heravi ${ }^{2}$; Shojaoddin Shayegh ${ }^{3}$; Ramtin Azar $^{4}$; Farshid Bastami ${ }^{4,}$ \\ ${ }^{1}$ Department of Periodontics, Dental School, Shahed University, Tehran, IR Iran \\ 2 Elderly Care Research Center, Nursing and Midwifery College, Shahed University, Tehran, IR Iran \\ 3 Department of Prosthodontics, Dental School, Shahed University, Tehran, IR Iran \\ ${ }^{4}$ Dental School, Shahed University, Tehran, IR Iran \\ ${ }^{*}$ Corresponding author: Farshid Bastami, Dental School, Shahed University, Italy St., Vesal Ave., Keshavarz Blv., Tehran, IR Iran. Tel: +98-2188959210, Fax: +98-2188954915, E-mail: \\ farshid_bst@yahoo.com
}

Received: June 7, 2013; Accepted: July 1, 2013

\begin{abstract}
Background: Clinicians and policymakers are recognizing the importance of measuring health-related quality of life (HRQL) to inform patient management and policy decisions.

Objectives: With regard to relation between cultural differences and quality of life (QoL), in this study the QoL of Iranian patients with conventional complete denture and implant-retained overdenture prosthesis according to the increasing penchant of use of implant-retained overdenture prosthesis and lack of studies in this field in our country, has been compared.

Patients and Methods: In this cross-sectional study, cluster sampling was conducted in two stages and 90 edentulous patients (45 patients with conventional complete dentures for two jaws (CD group) and 45 patients with a conventional maxillary complete dentures and implant-retained mandibular denture based on two intercanine implants (IOD group)) with the age of above 35 years were selected according to our inclusion criteria. After obtaining permission from the patients, who had been treated at the selected healthcare and medical treatment centers of Tehran, basic information was obtained by a checklist, including gender, age, educational status and housing status; and QoL was measured by a questionnaire of oral health impact profile (OHIP-20).

Results: In the group of IOD, 55.6\% of the patients had "good" QoL vs. $46.7 \%$ of the patients in CD group. Three patients (6.6\%) in both groups had "poor" QoL. No significant relationship was observed between demographic factors and different dimensions of QoL, except the average of "psychological disability" in the patients of CD group, which was higher in women.

Conclusions: According to this study results, implant-retained overdenture prosthesis provided better QoL for Iranian patients.
\end{abstract}

Keywords:Quality of Life; Health; Dental Implant; Overdenture Prosthesis; Complete Denture

\section{Background}

Nowadays, life expectancy is increasing because of recent health promotion (1). Unfortunately, due to systemic diseases, home oral care low compliance, especially in smokers, lack of periodic visit to the dentist and high insurance franchise completely edentulous patients are increasing among not only in the elderly, but also the youth in Iran (2). Therefore, the need of dental treatments is increasing. After the theory of Osseo Integration, introduced in 1982 in Toronto Conference, the use of implant overdenture prosthesis gradually became prevalent (3); thus, two therapeutic options can be considered for edentulous patients: conventional complete denture (CD) and implantretained overdenture prosthesis (IOD). Since "health" was introduced as "a complete state of physical, mental, and social well-being and not just the absence of disease" by the World Health Organization (4), progressively, the concept of Quality of Life (QoL) has been turned into one of the most important debatable issues in clinical research- es (5). In most countries, presented results are used by the patient to evaluate the effect of treatments, scientifically and clinically. One of the strongest and most efficient tools to measure QoL is Oral Health Impact Profile (OHIP) which is derived from a model of oral health status (6-10). OHIP-20 is a 20-item questionnaire, which describes the effect of oral health status on daily life performance and social relations based on seven dimensions related to the QoL. These dimensions include: physical pain, functional limitation, physical disability, psychological discomfort, social disability, psychological disability and handicap (11-15). It is striking that relationship between cultural differences and QoL was revealed for other medical conditions $(16,17)$; aspects of QoL in different cultural groups, in fact, may be different (18). Recently, cultural differences and their relationship to QoL of CD and IOD patients compared with OHIP-20 questionnaire was demonstrated by an international multicenter study (19). 


\section{Objectives}

Due to the expanded use of dental implants in Iran and lack of studies in this field in this country with regard to Iranian's cultural differences with previous studies' participants, the aim of this study was to compare the Oral Health-related Quality of Life of Iranian patients using CD and IOD, and to study their relationship with sociodemographic factors such as education, housing status, and gender of patients.

\section{Materials and Methods}

\subsection{Data Collection}

Two set of questions were used. The first was a checklist included patients' demographic information which contains the following information: $1-$ Gender: male and female, 2- Age, 3- Education: Below Diploma, Diploma, Bachelor and Master and above. 4- Housing Status: rented, private. The second one was OHIP-20 questionnaire. After requesting and obtaining permission from designer of OHIP-20, Slade G, this questionnaire was translated in Forward-Backward approach $(20,21)$. Moreover, the content validity analysis was used to determine the scientific validity of the questionnaire (22); in addition, its Internal Consistency was measured with interpreting Cronbach's Alpha coefficient (23). Also, validity and reliability of the Persian version of the questionnaire has been approved.

\subsection{Study Population}

In this cross-sectional study, cluster sampling was conducted in two stages. Randomly selected cluster was made in the first stage, while selection of all cluster members eligible for inclusion was made in the second stage. After obtaining approval from medical centers, selected healthcare and medical treatment centers of Tehran, patients, who met the inclusion criteria, were selected and examined clinically and radio-graphically. Then, OHIP-20 was completed. We enrolled 90 edentulous patients regarding our inclusion criteria: (Table 1) 45 patients with conventional complete dentures for two jaws (CD group) and 45 patients with a conventional maxillary complete denture and implant-retained mandibular overdenture prosthesis, based on two intercanine implants (IOD group).

\subsection{Measures and Study Design}

Items of the OHIP-20 were scored on six-point Likert scales: never, rarely, occasionally, often, very often, all the time, which were assigned numerical values between one (never) and six (all the time). In fact, if the patient obtains lower score; he or she enjoys life better, i.e. higher QoL (24$26)$. Scores were categorized in three ranges: 20-40, 40100 and 100-120 which showed good, moderate and poor
QoL, respectively. Normality of data distribution in each group was first confirmed with Kolmogorov-Smirnov Fit Goodness Test (KSFGT); then independent samples t-test was used to study difference of QoL between each treatment group, and ANOVA was used to study relationship between educational level and QoL. Moreover, nonparametric Mann-Whitney-U Test was used to study relationship between housing status and different dimensions of QoL in each treatment group. All statistical analyses were performed by SPSS version 13. P values less than 0.05 was considered as significant.

\section{Results}

In this study, Cronbach's Alpha coefficient was 0.818, and validity and reliability of the Persian version of the questionnaire was confirmed. In IOD Group, 55.6\% of patients ( $n=25)$ had "good" QoL, and 37.8\% ( $n=17)$ had "moderate" QoL. On the other hand, in CD Group, $46.7 \%$ of patients (n = 21) had "good" QoL, and 46.7\% (n=21) had "moderate" QoL. Only three patients in each groups (6.7\%) had scores in "poor" QoL range. The total average score of seven dimensions related to QoL, measured by OHIP-20, was 43.82 \pm 15.95 and $48.64 \pm 18.44$ for IOD and CD groups, respectively. While for IOD group, the mean score in functional limitation was obtained $6.89 \pm 3.48$, in physical pain 8.22 \pm 3.25 , in psychological discomfort $6.82 \pm 7.97$, in physical disability $8 \pm 3.43$, in psychological disability $4.16 \pm$ 1.98 , in social disability $6.04 \pm 2.61$ and in handicap 3.69 \pm 1.79 , for CD group, the mean score in functional limitation was found $8.11 \pm 3.93$, in physical pain $10 \pm 4.63$, in psychological discomfort $6.78 \pm 3.18$, in physical disability $10.42 \pm 5.68$, in psychological disability $4.22 \pm 1.86$, in social disability $5.11 \pm 2.25$, and in handicap $4.00 \pm 1.921$. In studying relationship between socio-demographic factors and different dimensions of QoL, no significant relationship was observed with regard to level of education $(P>0.05)$ (Table 2) and housing status $(\mathrm{P}>0.05)$ (Table 3 ). No significant difference was also found between gender and QoL in IOD patients $(\mathrm{P}>0.05)$. With regard to the $C D$ patients,

\begin{tabular}{l}
\hline Table 1. Inclusion Criteria of the Studied Patients \\
\hline Inclusion Criteria of the Studied Patients \\
\hline Passing at Least One Year of Treatment \\
Complete Edentulism for at Least 3 Years \\
Lack of Confirmed Mental Illness \\
No Alcoholism, Excessive Obesity and Smoking More Than One \\
Pack Per Day \\
Lack of Temporomandibular Joint Dysfunction \\
Absence of Uncontrolled Systemic Disease \\
Age of 35 Years and above \\
Ability to Speak Persian \\
\hline Residence in Tehran \\
Willingness to Participate in the Research \\
\hline
\end{tabular}


Semyari Het al.

Table 2. Oral Health Impact Profile-20 Scores in Different Levels of Education in Two Groups a,b

\begin{tabular}{|c|c|c|c|c|c|c|c|c|c|c|}
\hline & \multicolumn{5}{|c|}{ IOD Group $(n=45)$} & \multicolumn{5}{|c|}{$\operatorname{CD} \operatorname{Group}(n=45)$} \\
\hline & $\begin{array}{c}\text { Below Diploma } \\
\qquad(\mathbf{n}=\mathbf{1 1})\end{array}$ & $\begin{array}{c}\text { Diploma } \\
(\mathbf{n}=11)\end{array}$ & $\begin{array}{c}\text { Bachelor } \\
(\mathbf{n}=15)\end{array}$ & $\begin{array}{l}\text { Master } \\
(\mathbf{n}=\mathbf{8})\end{array}$ & PValue & $\begin{array}{c}\text { Below Diploma } \\
(\mathbf{n}=\mathbf{2 3})\end{array}$ & $\begin{array}{c}\text { Diploma } \\
(\mathbf{n}=12)\end{array}$ & $\begin{array}{l}\text { Bachelor } \\
(\mathbf{n}=7)\end{array}$ & $\begin{array}{l}\text { Master } \\
(\mathbf{n}=3)\end{array}$ & PValue \\
\hline $\begin{array}{l}\text { Functional } \\
\text { Limitation }\end{array}$ & $6.72 \pm 2.053$ & $7.81 \pm 5.05$ & $5.66 \pm 2.41$ & $8.12 \pm 3.94$ & 0.03 & $8.34 \pm 3.60$ & $8.83 \pm 4.74$ & $5.42 \pm 3.64$ & $9.66 \pm 0.57$ & 0.24 \\
\hline Physical Pain & $6.81 \pm 2.60$ & $8.91 \pm 2.98$ & $9.06 \pm 4.18$ & $7.62 \pm 1.84$ & 0.28 & $9.95 \pm 4.86$ & $10.33 \pm 4.55$ & $10.14 \pm 5.01$ & $8.66 \pm 4.16$ & 0.95 \\
\hline $\begin{array}{l}\text { Psychological } \\
\text { Discomfort }\end{array}$ & $4.18 \pm 2.13$ & $6.09 \pm 2.98$ & $6.66 \pm 3.01$ & $11.75 \pm 17.95$ & 0.22 & $6.26 \pm 2.84$ & $7.16 \pm 3.58$ & $7.57 \pm 3.20$ & $7.33 \pm 5.03$ & 0.74 \\
\hline $\begin{array}{l}\text { Physical } \\
\text { Disability }\end{array}$ & $7.00 \pm 2.75$ & $7.54 \pm 2.46$ & $8.66 \pm 4.87$ & $8.75 \pm 1.83$ & 0.57 & $10.04 \pm 6.04$ & $11.50 \pm 5.64$ & $9.28 \pm 5.58$ & $11.66 \pm 4.61$ & 0.82 \\
\hline $\begin{array}{l}\text { Psychological } \\
\text { Disability }\end{array}$ & $3.54 \pm 2.25$ & $4.09 \pm 1.70$ & $4.66 \pm 2.35$ & $4.12 \pm 2.99$ & 0.57 & $3.82 \pm 1.77$ & $3.91 \pm 1.37$ & $5.42 \pm 2.50$ & $5.66 \pm 1.15$ & 0.10 \\
\hline Social Disability & $5.18 \pm 1.99$ & $5.54 \pm 1.69$ & $6.80 \pm 3.64$ & $6.50 \pm 1.85$ & 0.38 & $4.52 \pm 1.78$ & $5.00 \pm 1.95$ & $6.71 \pm 3.54$ & $6.33 \pm 1.52$ & 0.10 \\
\hline Handicap & $2.81 \pm 1.16$ & $3.27 \pm 1.42$ & $4.53 \pm 2.26$ & $3.87 \pm 1.45$ & 0.08 & $3.47 \pm 1.30$ & $4.66 \pm 1.72$ & $4.42 \pm 3.50$ & $4.33 \pm 1.52$ & 0.31 \\
\hline Total & $36.27 \pm 8.62$ & $43.27 \pm 11.02$ & $46.06 \pm 18.80$ & $50.75 \pm 21.33$ & 0.23 & $46.43 \pm 17.61$ & $51.41 \pm 18.47$ & $49.00 \pm 25.05$ & $53.66 \pm 12.85$ & 0.85 \\
\hline
\end{tabular}

a Abbreviations: IOD, implant-retained overdenture; $C D$, complete denture.

$\mathrm{b}$ Data are presented as Mean \pm SD.

Table 3. Oral Health Impact Profile-20 Scores in Types of Housing Status in Two Groups a,b

\begin{tabular}{|c|c|c|c|c|c|c|}
\hline & \multicolumn{3}{|c|}{ IOD Group $(n=45)$} & \multicolumn{3}{|c|}{ CD Group $(n=45)$} \\
\hline & Rented $(n=5)$ & Private $(n=40)$ & P Value & $\operatorname{Rented}(\mathbf{n}=9)$ & Private $(n=36)$ & PValue \\
\hline $\begin{array}{l}\text { Functional Limi- } \\
\text { tation }\end{array}$ & $5.80 \pm 2.77$ & $7.02 \pm 3.56$ & 0.53 & $9.00 \pm 4.15$ & $7.88 \pm 3.89$ & 0.40 \\
\hline Physical Pain & $10.20 \pm 5.76$ & $7.97 \pm 2.82$ & 0.40 & $9.77 \pm 5.26$ & $10.05 \pm 4.53$ & 0.72 \\
\hline $\begin{array}{l}\text { Psychological } \\
\text { Discomfort }\end{array}$ & $5.80 \pm 3.11$ & $8.40 \pm 6.95$ & 0.95 & $6.44 \pm 3.43$ & $6.86 \pm 3.16$ & 0.79 \\
\hline $\begin{array}{l}\text { Physical Dis- } \\
\text { ability }\end{array}$ & $10.80 \pm 5.35$ & $7.65 \pm 3.03$ & 0.14 & $11.00 \pm 6.57$ & $10.27 \pm 5.52$ & 0.94 \\
\hline $\begin{array}{l}\text { Psychological } \\
\text { Disability }\end{array}$ & $4.80 \pm 3.34$ & $4.07 \pm 1.78$ & 0.90 & $3.44 \pm 1.50$ & $4.41 \pm 1.90$ & 0.22 \\
\hline Social Disability & $7.00 \pm 3.39$ & $5.92 \pm 2.52$ & 0.49 & $4.77 \pm 1.85$ & $5.19 \pm 2.35$ & 0.74 \\
\hline Handicap & $4.20 \pm 3.49$ & $3.62 \pm 1.53$ & 0.84 & $3.77 \pm 1.78$ & $4.05 \pm 1.97$ & 0.70 \\
\hline Total & $48.60 \pm 24.08$ & $43.22 \pm 14.96$ & 0.84 & $48.22 \pm 19.31$ & $48.75 \pm 18.50$ & 0.98 \\
\hline
\end{tabular}

a Abbreviations: IOD, implant-retained overdenture; CD, complete denture.

${ }^{b}$ Data are presented as Mean \pm SD.

Table 4. Oral Health Impact Profile -20 Scores in Types of Gender in Two Groups a,b

\begin{tabular}{lccccc}
\hline & \multicolumn{3}{c}{ IOD Group $(\mathbf{n}=\mathbf{4 5})$} & \multicolumn{2}{c}{ CD Group $(\mathbf{n}=\mathbf{4 5})$} \\
\cline { 2 - 6 } & Male $(\mathbf{n}=\mathbf{2 6})$ & Female $(\mathbf{n}=\mathbf{1 9})$ & P Value & Male $(\mathbf{n}=\mathbf{2 4})$ & Female $(\mathbf{n}=\mathbf{2 1})$ \\
\hline Functional Limitation & $7.07 \pm 3.41$ & $6.63 \pm 3.63$ & 0.67 & $8.83 \pm 3.82$ & $7.28 \pm 3.96$ \\
Physical Pain & $7.46 \pm 1.70$ & $9.26 \pm 4.45$ & 0.10 & $10.45 \pm 4.27$ & $9.47 \pm 5.05$ \\
Psychological Discomfort & $7.11 \pm 10.21$ & $6.42 \pm 3.16$ & 0.77 & $6.95 \pm 3.56$ & $6.57 \pm 2.74$ \\
Physical Disability & $7.76 \pm 2.30$ & $8.31 \pm 4.60$ & 0.60 & $11.37 \pm 5.82$ & $9.33 \pm 5.42$ \\
Psychological Disability & $3.84 \pm 1.51$ & $4.57 \pm 2.45$ & 0.22 & $4.75 \pm 1.77$ & $3.61 \pm 1.80$ \\
Social Disability & $5.73 \pm 2.35$ & $6.47 \pm 2.93$ & 0.35 & $5.37 \pm 2.56$ & $4.80 \pm 1.83$ \\
Handicap & $3.46 \pm 1.58$ & $4.00 \pm 2.05$ & 0.32 & $4.41 \pm 2.24$ & 0.48 \\
Total & $42.46 \pm 14.98$ & $45.68 \pm 17.41$ & 0.50 & $52.16 \pm 18.59$ & $4.52 \pm 1.36$ \\
\hline
\end{tabular}

a Abbreviations: IOD, implant-retained overdenture; CD, complete denture.

$\mathrm{b}$ Data are presented as Mean $\pm S D$. 
no significant relationship was observed between gender and other dimensions related to the QoL, except in psychological disability dimension. The mean psychological disability was higher in women $(\mathrm{P}<0.05)$ (Table 4).

\section{Discussion}

According to this study, the Oral Health-related Quality of Life of Iranian patients using mandibular 2-implant overdenture prosthesis and conventional complete denture was compared with regard to Iranian's sociodemographic factors influenced by Iranian cultures. The results revealed that $55.6 \%$ and $46.7 \%$ of individuals in IOD and CD groups have "good" QoL, respectively. Generally, in most studies, QoL in patients treated by the implant enjoyed more favorable conditions regardless of cultural differences (27-31). It was also shown that new conventional dentures are not well advised to meet patients' expectations (32). Although, the overall QoL mean score was $43.82 \pm 15.9$ and $48.64 \pm 18.44$ in IOD and $\mathrm{CD}$ groups, respectively, greater average distance between the two groups has been shown in previous studies in north America and Europe which were reported $66.1 \pm 28.08,35 \pm 15.94$ and $85.20 \pm 19.57$ for IOD group, compared with $89.3 \pm 40.42,47.84 \pm 22.6$ and $103.74 \pm 30.96$ for CD group respectively $(11,12,33)$. On the contrary, in one study in Ireland, the overall mean score of QoL was reported 65.9 and 40.5 in IOD and CD groups, respectively (13); which mean lower QoL in the IOD group than the CD group for receiving implant only in a jaw may not fully satisfy these patients completely. In the present study, in IOD Group, the highest level of QoL was observed in dimensions of handicap, which reveals feeling of less satisfaction from life because of denture-related problems, psychological disability, which manifests anger or embarrassment because of dental problems, social disability, which shows avoidance of participating in community and feeling less tolerable to the individuals because of denture problems, and psychological discomfort, which indicates anxiety and worries associated with denture-induced problems (12, 33). For example, Awad et al. reported the highest level of QoL in IOD patients with $6.7 \pm 2.12$ in handicap, 7.9 \pm 3.6 in psychological disability, $5.5 \pm 1.32$ in social disability and $7.09 \pm 4.27$ in psychological discomfort dimensions in North American patients (11).

Moreover, according this results, in IOD group, the lowest level of QoL was observed in the dimensions of physical disability, which reveals inability to eat and being upset from diet, physical pain, which unveils pain when chewing, existence of wounds in the mouth and annoying denture, and functional limitation, which displays problems in eating and feeling denture instability and looseness, which are in line with the last findings $(11,33)$. For instance, Heydecke et al. reported the lowest level of QoL among IOD patients with $8.07 \pm 3.099$ in physi- cal pain, $6.5 \pm 3.79$ in physical disability and $8.1 \pm 3.72$ in functional limitation dimensions (12). In this study, in CD group, the highest level of QoL was observed in dimensions of handicap, psychological disability, social disability, psychological discomfort, and the lowest level of QoL was observed in dimensions of physical pain, physical disability and functional limitation consistent with previous findings $(11,12,33)$. Generally, mean QoL in one study is higher than the present findings, could be due to the use of OHIP-49 questionnaire (11). In one randomized-controlled trial study, which Groningen Activity Restriction Scale-Dentis (GARS-D) was used as QoL measurement tool for CD and IOD patients, QoL was reported equal in both groups (34). Similar findings obtained from other studies demonstrated that OHIP is more effective and accurate than other measurement tools of QoL and diagnoses differences between various treatments. However, significant difference was not observed between general health of IOD and CD patients in different studies $(12,13)$. Furthermore, oral health status is independent from general health status to some extent, and it has been shown that OHIP is more highly dependent to oral health conditions than the Short Form Health Survey (SF-36) (35). Therefore, measurement of general QoL of patients was not carried out in the present study. Moreover, in one study, which was conducted to examine the effect of mandibular ridge height on the perceptions of IOD and CD patients, no significant relationship was observed between these two factors; hence, patients' ridge height was not measured as a condition of entry to the research in the present study (36). According to the results, there was not any significant relationship between QoL and both educational level and housing status. In other words, mean of OHIP different dimensions in each four groups of different educational levels (Below Diploma, Diploma, Bachelor and Graduate and above) were equal with each other, and the average different OHIP dimensions were equal to the rented and owned housing status in both groups. Other results, similarly, did not report any significant relationship between demographic factors and QoL, which confirm these findings $(12,13)$. Furthermore, there was no significant relationship between gender and QoL in both groups except in psychological disability aspect in CD group. In fact, the QoL mean score in psychological disability aspect was more in women than men; still, other results stated that demographic factors are effective as nearly as 31\% in the score rate of QoL after treatment, which was not is accordance to our results (11).

According to these results, implant-retained mandibular denture based on two intercanine implants had favorable QoL among Iranian patients regardless of cultural differences with other countries studies' participants. Measurement of QoL also provides well-grounded documents available for patients and clinicians, which can estimate effect of treatment interactions in improvement of QoL of patients. 


\section{Acknowledgements}

We express our deepest thanks to both head and staff of medical centers, who helped us to find the eligible patients.

\section{Authors Contributions}

Study concept and design: Semyari and Heravi; acquisition of data: Heravi, Shayegh, Azar and Bastami; snalysis and interpretation of data: Semyari, Heravi, Azar and Bastami; drafting of the manuscript: Bastami; critical revision of the manuscript for important intellectual content: Semyari, Heravi, Shayegh, Azar and Bastami; statistical analysis: Azar and Bastami.

\section{References}

1. Truhlar RS, Casino AJ, Cancro JJ. Treatment planning of the elderly implant patient. Dent Clin North Am. 1997;41(4):847-61.

2. Ahmadzadeh A, Fereidoonpoor N. [Comparison of Retentive Strength in Variable Attachment Systems in Implant-Supported Overdenture]. J Mashhad Dent School.2013;36(4):259-69.

3. Albrektsson T, Zarb G, Worthington P, Eriksson AR. The long-term efficacy of currently used dental implants: a review and proposed criteria of success. Int J Oral Maxillofac Implants.1986;1(1):11-25.

4. World Health Organization.. Constitution of the World Health Organization. Geneva Switzerland: WHO; 1984. Available from: http:/ www.who.int/governance/eb/who_constitution_en.pdf.

5. Torrance GW. Utility approach to measuring health-related quality of life. J Chronic Dis. 1987;40(6):593-603.

6. Slade GD, Spencer AJ. Development and evaluation of the Oral Health Impact Profile. Community Dent Health. 1994;11(1):3-11.

7. Slade GD, Strauss RP, Atchison KA, Kressin NR, Locker D, Reisine ST. Conference summary: assessing oral health outcomes--measuring health status and quality of life. Community Dent Health. 1998;15(1):3-7.

8. Locker D, Allen F. What do measures of 'oral health-related quality of life' measure? Community Dent Oral Epidemiol. 2007; 35(6):401-11.

9. Hebling E, Pereira AC. Oral health-related quality of life: a critical appraisal of assessment tools used in elderly people. Gerodontology. 2007;24(3):151-61.

10. Thomason JM, Heydecke G, Feine JS, Ellis JS. How do patients perceive the benefit of reconstructive dentistry with regard to oral health-related quality of life and patient satisfaction? A systematic review. Clin Oral Implants Res. 2007;18 Suppl 3:168-88.

11. Awad MA, Locker D, Korner-Bitensky N, Feine JS. Measuring the effect of intra-oral implant rehabilitation on health-related quality of life in a randomized controlled clinical trial. J Dent Res. 2000;79(9):1659-63.

12. Heydecke G, Locker D, Awad MA, Lund JP, Feine JS. Oral and general health-related quality of life with conventional and implant dentures. Community Dent Oral Epidemiol. 2003;31(3):161-8.

13. Allen PF, McMillan AS. A longitudinal study of quality of life outcomes in older adults requesting implant prostheses and complete removable dentures. Clin Oral Implants Res. 2003;14(2):173-9.

14. John MT, Slade GD, Szentpetery A, Setz JM. Oral health-related quality of life in patients treated with fixed, removable, and complete dentures 1 month and 6 to 12 months after treatment. Int $J$ Prosthodont. 2004;17(5):503-11.

15. Allen PF, Thomason JM, Jepson NJ, Nohl F, Smith DG, Ellis J. A randomized controlled trial of implant-retained mandibular overdentures. J Dent Res. 2006;85(6):547-51.

16. Scott NW, Fayers PM, Aaronson NK, Bottomley A, de Graeff A Groenvold M, et al. The relationship between overall quality of life and its subdimensions was influenced by culture: analysis of an international database. J Clin Epidemiol. 2008;61(8):788-95.

17. Molzahn AE, Kalfoss M, Schick Makaroff K, Skevington SM. Comparing the importance of different aspects of quality of life to older adults across diverse cultures. Age Ageing. 2011;40(2):1929.

18. Kagawa-Singer M, Padilla GV, Ashing-Giwa K. Health-related quality of life and culture. Semin Oncol Nurs. 2010;26(1):59-67.

19. Awad MA, Rashid F, Feine IS, Overdenture Effectiveness Study Team C. The effect of mandibular 2-implant overdentures on oral health-related quality of life: an international multicentre study. Clin Oral Implants Res. 2014;25(1):46-51.

20. Beaton DE, Bombardier C, Guillemin F, Ferraz MB. Guidelines for the process of cross-cultural adaptation of self-report measures. Spine (Phila Pa 1976). 2000;25(24):3186-91.

21. van der Meulen MJ, John MT, Naeije M, Lobbezoo F. The Dutch version of the Oral Health Impact Profile (OHIP-NL): Translation, reliability and construct validity. BMC Oral Health. 2008;8:11.

22. Yamazaki M, Inukai M, Baba K, John MT. Japanese version of the Oral Health Impact Profile (OHIP-J). J Oral Rehabil. 2007;34(3):159-68.

23. Bland JM, Altman DG. Statistics notes: Cronbach's alpha. Br Med J. 1997;314(7080):572.

24. Locker D, Matear D, Stephens M, Jokovic A. Oral health-related quality of life of a population of medically compromised elderly people. Community Dent Health. 2002;19(2):90-7.

25. Awad MA, Lund JP, Shapiro SH, Locker D, Klemetti E, Chehade A, et al. Oral health status and treatment satisfaction with mandibular implant overdentures and conventional dentures: a randomized clinical trial in a senior population. Int J Prosthodont. 2003;16(4):390-6.

26. Forgie AH, Scott BJ, Davis DM. A study to compare the oral health impact profile and satisfaction before and after having replacement complete dentures in England and Scotland. Gerodontology. $2005 ; 22(3): 137-42$.

27. Emami E, Heydecke G, Rompre PH, de Grandmont P, Feine JS Impact of implant support for mandibular dentures on satisfaction, oral and general health-related quality of life: a metaanalysis of randomized-controlled trials. Clin Oral Implants Res. 2009;20(6):533-44.

28. Turkyilmaz I, Company AM, McGlumphy EA. Should edentulous patients be constrained to removable complete dentures? The use of dental implants to improve the quality of life for edentulous patients. Gerodontology. 2010;27(1):3-10.

29. Assuncao WG, Barao VA, Delben JA, Gomes EA, Tabata LF. A comparison of patient satisfaction between treatment with conventional complete dentures and overdentures in the elderly: a literature review. Gerodontology. 2010;27(2):154-62.

30. Albaker AM. The oral health-related quality of life in edentulous patients treated with conventional complete dentures. Gerodontology. 2013;30(1):61-6.

31. Thomason JM. The use of mandibular implant-retained overdentures improve patient satisfaction and quality of life. J Evid Based Dent Pract. 2012;12(3 Suppl):182-4.

32. Heydecke G, Thomason JM, Awad MA, Lund JP, Feine JS. Do mandibular implant overdentures and conventional complete dentures meet the expectations of edentulous patients? Quintessence Int. 2008;39(10):803-9.

33. Heydecke G, Thomason JM, Lund JP, Feine JS. The impact of conventional and implant supported prostheses on social and sexual activities in edentulous adults Results from a randomized trial 2 months after treatment. J Dent. 2005;33(8):649-57.

34. Bouma J, Boerrigter LM, Van Oort RP, van Sonderen E, Boering G. Psychosocial effects of implant-retained overdentures. Int J Oral Maxillofac Implants. 1997;12(4):515-22.

35. Sischo L, Broder HL. Oral health-related quality of life: what, why, how, and future implications. J Dent Res. 2011;90(11):1264-70.

36. Kimoto K, Garrett NR. Effect of mandibular ridge height on patients' perceptions with mandibular conventional and implant-assisted overdentures. Int J Oral Maxillofac Implants. 2005;20(5):762-8. 